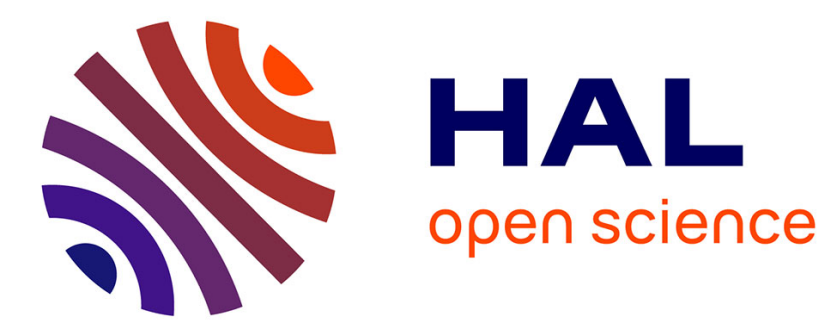

\title{
Rendre le patient transplanté rénal acteur de sa santé
} Johanna Rabiller, Nicolas Clere, Céline Onno

\section{To cite this version:}

Johanna Rabiller, Nicolas Clere, Céline Onno. Rendre le patient transplanté rénal acteur de sa santé. Actualités Pharmaceutiques, 2019, 58, pp.33 - 38. 10.1016/j.actpha.2019.01.017 . hal-03485649

\section{HAL Id: hal-03485649 \\ https://hal.science/hal-03485649}

Submitted on 20 Dec 2021

HAL is a multi-disciplinary open access archive for the deposit and dissemination of scientific research documents, whether they are published or not. The documents may come from teaching and research institutions in France or abroad, or from public or private research centers.
L'archive ouverte pluridisciplinaire HAL, est destinée au dépôt et à la diffusion de documents scientifiques de niveau recherche, publiés ou non, émanant des établissements d'enseignement et de recherche français ou étrangers, des laboratoires publics ou privés.

\section{다)(1) $\$$}

Distributed under a Creative Commons Attribution - NonCommercial| 4.0 International 
Dochead dossier

Sous-dochead La greffe rénale

\title{
Rendre le patient transplanté rénal acteur de sa santé à l'officine
}

\author{
Johanna Rabiller ${ }^{\mathrm{a}}$ \\ Pharmacienne adjointe
}

Nicolas Clere ${ }^{\mathrm{b}, *}$

Maître de conférences des Universités en pharmacologie

\section{Céline Onnoc}

Médecin néphrologue

aPharmacie Gambart-Dhaine, 72 rue des Sables, 85300 Challans, France

${ }^{\text {b}}$ Faculté de santé, Département pharmacie, Université d'Angers, 16 boulevard Daviers, 49045 Angers, France

'Centre hospitalier universitaire d'Angers, Service de néphrologie, dialyse, transplantation, 4 rue Larrey, 49933 Angers cedex 9, France

*Auteur correspondant.

Adresse e-mail : nicolas.clere@univ-angers.fr (N. Clere).

Résumé

L'accompagnement du patient transplanté rénal s'avère complexe du fait de l'importance, pour lui, d'adhérer au traitement et d'intégrer sa maladie à sa vie quotidienne. Les équipes officinales sont en première ligne pour l'accompagner, le rassurer, le conseiller, voire l'orienter. L'éducation thérapeutique occupe une place privilégiée dans cette prise en charge.

(C) 2019

Mots clés - accompagnement ; éducation thérapeutique ; greffe ; immunosuppresseur ; observance ; officine

Summary à venir 
Keywords à venir

Le patient transplanté rénal est atteint d'une maladie chronique. Il est soumis à une prise en charge spécifique en post-greffe immédiat : traitement immunosuppresseur et prophylaxie antibiotique, antifongique, voire antivirale. Après la phase initiale de prise en charge qui est organisée en milieu hospitalier, il doit continuer à prendre son traitement immunosuppresseur pendant toute la durée de survie du greffon et se plier à un suivi régulier reposant sur des consultations externes, très rapprochées au début, et parfois de courts séjours hospitaliers.

Le patient greffé est parfois atteint d'autres maladies chroniques préexistantes comme le diabète, l'épilepsie, l'asthme, les cardiopathies... De ce fait, il s'agit d'un malade polymédicamenté soumis à des traitements pouvant générer des effets indésirables et dont les molécules, les doses et les posologies peuvent être modulées. Des difficultés de prise en charge sur le plan clinique, thérapeutique, économique et psychosocial peuvent également être observées.

\section{T1 Les besoins des patients}

Les patients greffés expriment certains ressentis ou problématiques également retrouvés chez les sujets atteints d'autres maladies chroniques, tels que la lassitude de la prise d'un traitement médicamenteux au long cours.

\section{T2 Besoin de connaissances}

TEG1 La plupart des patients greffés ne connaissent pas les noms des médicaments qui leur sont prescrits ainsi que leurs rôles [1], ce qui montre qu'il n'est pas nécessaire de disposer de connaissances approfondies pour adhérer à son traitement. La "connaissance" ou le savoir théorique de la maladie et des traitements, contrairement à ce que pensent les soignants, n'est d'ailleurs pas identifiée comme un déterminant incontournable de l'adhésion [2]. Une étude concernant des adolescents diabétiques a montré que les patients qui avaient une connaissance correcte de leurs traitements et de la manière de les administrer étaient observants, tandis que ceux qui avaient une très faible connaissance ou, au contraire, une connaissance très poussée de leur maladie (concernant les conséquences à long terme, le pronostic) ne l'étaient pas [3].

TEG1 Au cours des échanges avec le patient, il semble nécessaire d'expliquer systématiquement le rôle des médicaments et ceci, toujours avec des mots simples. En effet, tout professionnel de santé doit substituer au jargon médical un langage intelligible pour tous. L'information doit également reposer sur l'emploi de termes mesurés, sans aller au-delà de ce que le malade veut savoir ou peut entendre. Aussi, il ne paraît pas primordial de répéter systématiquement l'indication de chaque traitement si la demande n'émane pas du malade lui-même, d'autant plus si la méconnaissance du traitement ne semble pas avoir d'impact négatif sur la prise médicamenteuse.

TEG1 Les informations concernant la maladie, sa prise en charge, son traitement spécifique et les précautions à prendre ne sont pas toujours assimilées par le patient [4]. Certains malades font ainsi part de représentations erronées du traitement antirejet, du rejet lui-même et/ou des traitements associés ne concernant pas directement le greffon. Par exemple, ils assimilent fréquemment le rejet à un retour en dialyse (par mort de l'organe transplanté). Ils doivent prendre conscience qu'il s'agit 
d'une complication curable, sans qu'il faille pour autant banaliser la situation (séquelles concernant le fonctionnement du greffon, altération de la durée de survie du rein). Toutefois, il est primordial qu'ils connaissent les signes cliniques caractérisant le début de rejet du greffon (œdèmes, prise de poids...). En effet, des pathologies silencieuses associées, comme l'hypertension artérielle (HTA) et le diabète, peuvent avoir un impact sur le rein. Par ailleurs, l'absence de symptômes a des conséquences : le patient peut ne pas se sentir "malade" et être également dans l'incapacité d'évaluer l'efficacité de ses traitements. Le risque est qu'il soit non-observant à des mesures thérapeutiques qui présentent, de plus, des inconvénients visibles, voire des effets secondaires [4]. TEG1 L'observance peut être améliorée par la création d'un "symptôme" : auto-contrôle glycémique ou tensionnel, par exemple. À cet égard, il faut rappeler que tous les médicaments prescrits de manière ponctuelle, dont l'ordonnance ne provient pas forcément du néphrologue, sont importants. Les antibiotiques, par exemple, sont destinés à combattre l'infection susceptible d'être générée par l'affaiblissement des défenses de l'organisme. En effet, le traitement antirejet peut générer des infections, d'où la nécessité de respecter le nombre de prises et la durée de l'antibiothérapie mais aussi de réagir en cas de symptômes spécifiques, comme la fièvre (capacité d'autosoin).

TEG1 Alors que les patients atteints de maladies rénales chroniques sont souvent traités pour d'autres pathologies, les études sur l'observance ne portent généralement que sur une seule maladie et ses traitements. Une analyse qualitative conduite auprès de 20 personnes âgées montre qu'elles établissent des priorités entre leurs traitements et leurs pathologies. Par exemple, une femme de 75 ans atteinte de huit comorbidités et prenant dix médicaments favorise son traitement hypertenseur par peur de l'infarctus. Dans cette étude, le facteur commun qui influence les choix des participants par rapport à la hiérarchisation de leurs maladies est la peur du plus grand risque. Les patients prennent ainsi en premier lieu les traitements concernant les pathologies les exposant à un grave danger si les symptômes n'en sont pas contrôlés. L'intérêt de cette étude est de montrer que les personnes âgées cumulant plusieurs pathologies créent leur propre arbre décisionnel [5]. TEG1 Afin de bannir les idées reçues sur le régime alimentaire à adopter après une transplantation rénale, une consultation diététique peut s'avérer utile. La diététicienne expliquera au patient qu'il convient de surveiller principalement le sel, distinguera les aliments riches en sel, évaluera sa consommation journalière en sodium et lui apprendra comment cuisiner moins salé en lui proposant des recettes adaptées.

TEG1 Pour permettre au patient de s'approprier son traitement, il pourrait être intéressant de proposer des entretiens dans l'espace de confidentialité de l'officine. Cela nécessiterait de préparer au préalable des petits dessins plastifiés représentant les organes soignés en fonction de tel médicament ou action sur un paramètre biologique donné : image $d^{\prime}$ un tensiomètre pour les traitements contre l'HTA, d'un rein pour les médicaments concernant la greffe, du cœur pour le traitement post-infarctus, d'un virus dans le cadre de la lutte contre les infections... À cette occasion, les médicaments prescrits seraient déballés (blister visible). Le patient, actif, pourrait ainsi placer chaque médicament sur l'un des dessins proposés en expliquant son choix (exploration des représentations, des croyances et des connaissances).

TEG1 Concernant les complications de la greffe, un échange reposant sur l'utilisation de cartes décisionnelles (fièvre, contexte de gastro-entérite...) pourrait être proposé. Le but serait de développer les compétences décisionnelles du patient : par exemple, interpréter des symptômes comme un signe d'alerte, décider de venir consulter ou d'appeler un médecin. 
TEG1 Réévaluer les moments de prise des médicaments, en particulier de la corticothérapie, est important. La clarté d'une ordonnance favorise la compréhension de la prise médicamenteuse du patient et donc son observance, mais permet aussi d'éviter toute erreur de délivrance par le pharmacien. En 2003, une étude descriptive transversale ayant porté sur 2586 ordonnances médicales a été réalisée dans la région de Sousse en Tunisie. La qualité de leur rédaction a été évaluée à travers deux paramètres : la lisibilité et les informations contenues. En tout, $36 \%$ des ordonnances renfermaient moins de $50 \%$ des items retenus, $25 \%$ étaient totalement illisibles tandis que la qualité de la rédaction était considérée bonne dans seulement $14 \%$ des cas [6]. Concernant les corticoïdes, le médecin doit "baliser" les moments de prise en inscrivant quelle dose est prise à telle date plutôt que d'inscrire les doses sur une durée. Par ailleurs, il faut toujours garder à l'esprit que des patients peuvent éprouver des difficultés pour couper les comprimés.

TEG1 II a été montré que l'observance était améliorée lorsque les patients participaient au processus de décision thérapeutique dans le cas d'une pathologie chronique, comme le psoriasis (dermatose inflammatoire chronique). Ils peuvent notamment exprimer une préférence pour l'une ou l'autre des formulations galéniques disponibles (gel, pommade, crème) selon leur facilité d'application, odeur, couleur ou caractère plus ou moins salissant. Il a été montré que cette implication avait un impact sur l'observance et l'évolution thérapeutique. Le praticien et le pharmacien doivent donc s'enquérir des préférences du patient pour telle ou telle formulation galénique [7].

TEG1 La prise en charge des effets indésirables participe à favoriser l'adhésion du patient à son traitement. Il importe de tenir compte des effets propres au patient et de proposer des médicaments ou des règles hygiéno-diététiques adaptés pour y faire face.

\section{T2 Besoins organisationnels}

TEG1 Les patients greffés utilisent fréquemment un pilulier afin de respecter les moments de prise des médicaments et emporter avec eux leur traitement lorsqu'ils sont en déplacement (travail, consultations médicales, restaurant, voyage).

TEG1 Un plan de prise médicamenteux peut être proposé aux patients. Certains éprouvent des difficultés à s'adapter aux doses régressives de corticoïdes. Lors de l'initiation du traitement immunosuppresseur, il est important de questionner préalablement la personne sur les traitements qu'elle suivait avant la greffe. Si certains d'entre eux sont à nouveau prescrits en période post-greffe et que les modalités de leur administration avant la greffe étaient appropriées, il faut rassurer le patient : ces dernières sont inchangées, ce qui sera moins perturbant pour lui et s'avérera plus efficace car correspondant aux habitudes de vie.

TEG1 Certains patients font part de circonstances particulières compliquant la prise de leur traitement antirejet à un moment donné. Pour les aider, I'utilisation d'une balance décisionnelle peut être intéressante : quel risque ai-je à ne pas prendre mon traitement ? Comment dois-je faire pour prendre mon médicament malgré la distance ? Comment savoir si la décision que je prends est la bonne ? D'autres interrogations peuvent survenir : quelle conduite dois-je tenir en cas d'oubli d'un comprimé sur une journée ? Comment dois-je m'organiser pour que cela ne se reproduise plus ? Dans le cas où l'oubli du traitement antirejet est effectué sur une journée, il convient d'expliquer au patient que celui-ci doit contacter son néphrologue qui pourra le renseigner sur la conduite à tenir. TEG1 Certains patients peuvent ressentir le besoin de réorganiser leurs horaires de travail en cas de difficulté : fatigue du fait des contraintes horaires par exemple. 
TEG1 Afin de faciliter la reconnaissance des médicaments par le patient, une stratégie adéquate peut lui être proposée. Ainsi, au lieu de les déconditionner pour les placer directement dans les cases du pilulier, il a tout intérêt à les conserver dans leur plaquette thermoformée sous blister, sur lequel figure le nom de spécialité. Ceci est d'autant plus vrai pour les médicaments qui doivent être pris dès l'ouverture du blister (Prograf ${ }^{\circledR}$ par exemple). Dans le cas où un pilulier est quand même utilisé, sa préparation par le patient peut être réalisée aux côtés d'une infirmière ou du pharmacien, tout comme la rédaction d'un plan de prise médicamenteux.

\section{T2 Besoins d'ordre social}

TEG1 Le besoin de se reconstruire après la greffe, de se réinsérer professionnellement notamment, ne doit pas être sous-estimé. En effet, le fait d'être atteint d'une maladie chronique est susceptible de représenter un préjudice pour les transplantés rénaux. Ne pas pouvoir poursuivre son activité professionnelle entraîne un isolement social et une perte de l'estime de soi.

TEG1 Les patients devraient pouvoir évoquer les difficultés auxquels ils sont confrontés après la greffe ou à distance, lors de la reprise du travail. Une assistante sociale peut, par exemple, les aider à gérer les lourdeurs administratives. Par ailleurs, l'intervention d'un médecin du travail peut être bénéfique pour ceux qui continuent à travailler malgré la dialyse.

\section{T2 Besoins d'ordre émotionnel}

TEG1 Les personnes greffées ressentent parfois une profonde angoisse, sans compter que l'équilibre au sein de la famille est souvent profondément bouleversé par la maladie, ce qui génère un stress important chez chacun de ses membres, risquant d'entraîner des complications psychologiques qui, à leur tour, peuvent influencer les relations familiales [8].

TEG1 L'intervention d'un psychologue peut contribuer au succès de la prise en charge de la maladie chronique et de ses conséquences psychologiques : culpabilité, sentiment de dette envers le donneur, angoisse liée à l'adoption d'un organe étranger, peur du rejet... [9].

\section{T1 Le rôle du pharmacien à l'officine}

Le pharmacien d'officine dispense les médicaments prescrits, conseille sur leur bon usage et met en garde contre la survenue possible d'effets indésirables. À ceci s'ajoutent l'information sur la maladie, le soutien psychosocial et le suivi régulier du patient et de son entourage. L'intervention de ce professionnel de proximité, via l'aide à la compréhension de la maladie et des traitements, l'apprentissage des techniques de prise et d'autosurveillance ainsi que le soutien quotidien, contribue donc à améliorer l'adhésion du malade à son traitement.

L'implication de l'officinal au sein d'une équipe de soins et/ou d'un réseau ville-hôpital peut s'avérer intéressante en transplantation rénale. Tous les intervenants doivent travailler ensemble et coordonner leurs actions. Cela nécessite que les médecins, pharmaciens et autres soignants soient capables de se positionner comme partenaires afin de construire, autour du patient, une véritable chaîne de soins et de prévention. Le maillage officinal apporte, dans ce cadre, une vraie valeur ajoutée dans la prise en charge des patients atteints de maladie chronique. 
Toutefois, même un pharmacien d'officine qui n'est pas intégré dans un réseau de soins peut aider le patient à acquérir des compétences d'adaptation et d'autosoin.

\section{T2 Informer le patient sur sa pathologie et les traitements}

TEG1 Pour adhérer à la proposition du traitement, le patient doit a priori comprendre a minima ce qui lui arrive, quels sont les médicaments proposés, comment ceux-ci fonctionnent et quels en sont les bénéfices et les risques. Les explications données par le pharmacien contribuent à renforcer les messages positifs déjà prodigués au patient par les différents professionnels de santé rencontrés tout au long de son parcours de soins [10]. Cette intervention nécessite le recours à des outils et documents adaptés à chaque contexte.

TEG1 Afin d'expliquer le rôle des différents médicaments prescrits, une planche de traitements remise par le pharmacien d'officine et complétée avec le patient peut s'avérer utile (figure 1). L'utilisation de pictogrammes propres à chaque pathologie favorise la compréhension.

TEG1 Des fiches conseils personnalisées permettent de renseigner plus avant le patient sur son traitement médicamenteux, les effets secondaires éventuels et les conduites à tenir en cas de symptômes spécifiques. Le pharmacien peut, pour les élaborer, s'appuyer sur les outils proposés par le Comité d'éducation sanitaire et sociale de la pharmacie française (Cespharm) [11]. Les documents diffusés doivent être commentés lors de leur remise au patient.

TEG1 L'apprentissage des techniques d'utilisation de certains médicaments est fondamental afin d'aider le patient à adhérer à son traitement, améliorer sa qualité de vie mais aussi assurer une bonne efficacité des médicaments.

TEG1 L'organisation pratique de l'administration des médicaments repose tout d'abord sur la négociation d'un plan de prise avec le patient, intégrant ses contraintes et habitudes de vie. Celui-ci doit définir la gestion domestique et la manipulation pratique des médicaments :

l'approvisionnement (lors de la sortie de l'hôpital ou d'un départ en vacances) mais aussi les pratiques de rangement, de conservation, de transport, de déconditionnement ou de fractionnement. Les décalages et les oublis de prise doivent également être abordés.

TEG1 Le pharmacien d'officine peut proposer de construire un plan de prise médicamenteux sur la journée ou la semaine, en négociant en particulier avec le malade l'organisation concernant les médicaments qui doivent être administrés à des heures plus ou moins fixes (figure 2). Il est nécessaire d'essayer d'aménager les horaires de prise en fonction des problématiques de vie, en définissant des points de repère : le départ au travail, le moment où il faut emmener les enfants à l'école, le passage de l'infirmière pour les soins... Les malades ont besoin d'être aidés dans les aspects pratiques de la vie quotidienne. Dans le cas où un patient éprouve des difficultés à s'adapter aux doses régressives de corticoïdes, un tableau peut être réalisé par le pharmacien et complété avec lui (figure 3). Par ailleurs, l'usage du pilulier ou du semainier s'avérant souvent utile aux patients polymédicamentés, le pharmacien peut également proposer ce dispositif lors de la délivrance des traitements.

\section{T2 Assurer la réception anticipée des ordonnances}

TEG1 La réception anticipée des ordonnances des malades à l'officine permet d'assurer la continuité des soins. Dès lors que le pharmacien d'officine est intégré dans un réseau de soins, le prescripteur (généraliste ou spécialiste) peut faxer ou envoyer par messagerie sécurisée les ordonnances du patient directement à la pharmacie afin que le dispensateur commande à l'avance les traitements (immunosuppresseurs, anti-infectieux...). Le cas échéant, la transmission des 
ordonnances peut également se faire via le contact téléphonique entre le pharmacien d'officine et l'externe en pharmacie en stage dans le service hospitalier.

TEG1 Dans le cas où le médicament prescrit est substituable, il est préférable que le pharmacien d'officine soit fidèle à un laboratoire donné. En effet, les médicaments génériques présentent parfois de légères différences en termes de présentation (couleur, forme) et de dénomination, qui peuvent apparaître significatives aux yeux des patients, modifier leurs habitudes et être à l'origine d'erreurs et de confusion [12]. Le pharmacien doit noter systématiquement le nom du princeps sur le conditionnement primaire du générique et le nom du médicament substitué accompagné de la mention "substitué par" sur l'ordonnance.

TEG1 Le pharmacien d'officine doit identifier la forme médicamenteuse qui convient le mieux ou qui semble la plus adaptée au patient en tenant compte de ses éventuels troubles de la vision, problèmes de déglutition ou de manipulation du médicament. La galénique est en effet considérée comme un facteur clé dans l'observance du traitement. Le dispensateur doit prendre connaissance des différentes formes médicamenteuses disponibles sur le marché.

\section{T2 Élaborer des stratégies pour limiter l'inobservance}

TEG1 Au comptoir, le patient peut faire part de situations quotidiennes ou d'événements particuliers rendant la prise médicamenteuse difficile. Il est important d'explorer ces situations avec lui et de l'aider à faire en sorte qu'elles ne se reproduisent plus. C'est la technique de l'incident critique.

TEG1 Le pharmacien doit conseiller au patient d'avoir sur lui, lors de tout déplacement, une ordonnance (ancienne ou non) ainsi que sa carte Vitale avec laquelle il est possible d'accéder à son dossier pharmaceutique.

\section{T2 Être disponible et à l'écoute}

TEG1 Le pharmacien d'officine doit clairement se présenter comme un partenaire compétent et compréhensif du malade, un médiateur entre le système de soins et le quotidien de ce dernier : il s'agit de lui permettre, lors de la dispensation des traitements, d'évoquer ses craintes, doutes et représentations, même les plus irrationnelles, et d'offrir un véritable soutien. L'officinal doit donc engager le dialogue, s'assurer de bien comprendre la demande et d'y répondre.

TEG1 Lors de la délivrance des médicaments, le patient peut être amené à évoquer ses difficultés. $S$ 'il fait part, par exemple, d'effets indésirables interférant avec son quotidien, le pharmacien doit lui donner des conseils hygiéno-diététiques, voire lui proposer des médicaments symptomatiques. Dans le cas où ces effets indésirables sont susceptibles d'avoir un impact sur l'observance médicamenteuse, l'officinal doit contacter le médecin prescripteur afin de savoir s'il est possible de remplacer le médicament incriminé par un autre, une démarche également utile lorsque des réévaluations de prescription sont nécessaires. Il est tenu de déclarer ces effets au Centre régional de pharmacovigilance dont il dépend [13].

TEG1 Même un pharmacien qui n'intervient pas en éducation thérapeutique du patient (ETP) peut être amené à orienter un malade et/ou son entourage vers d'autres professionnels :

un psychologue, une diététicienne, une assistante sociale... Le patient peut éventuellement être orienté vers une association de patients, comme France rein [14] et Renaloo [15]. Ces associations de santé ont joué un rôle signifiant dans le développement de l'ETP comme l'a notamment montré une enquête française publiée en 2002 [16]. Leur but est d'informer leurs adhérents, de les conseiller 
pour surmonter les difficultés de la vie quotidienne, de les soutenir psychologiquement et de les aider à trouver un nouvel équilibre dans leur vie familiale, sociale et professionnelle. Ce type de structure permet aussi au malade de partager l'expérience d'autres patients et donne, aussi souvent que possible, des conseils aux familles des patients, souvent anxieuses, voire désemparées et épuisées.

\section{Encadré}

\section{Accueillir le patient transplanté dans un espace adapté pour un accompagnement optimal}

Afin de favoriser les échanges confidentiels, d'instaurer un climat de confiance et de neutralité bienveillante, il est impératif qu'un espace de confidentialité soit mis en place dans les pharmacies d'officine pratiquant l'éducation thérapeutique du patient (ETP) et les entretiens pharmaceutiques. Évoquant les patients "sensibles", $30 \%$ des pharmaciens titulaires et $26 \%$ des pharmaciens adjoints ont présenté les sujets transplantés comme des sujets difficiles à prendre en charge au comptoir, derrière les patients dépressifs, toxicomanes, cancéreux et dialysés [17] ${ }^{1}$. L'accompagnement du patient greffé est délicat, mais indispensable. Le pharmacien d'officine est le professionnel de santé de proximité par excellence grâce au maillage territorial, assurant une présence constante et accessible sans contrainte. II constitue, de ce fait, un interlocuteur privilégié du système de santé et une porte d'entrée dans le parcours de soins [18].

${ }^{1}$ Étude réalisée auprès de 47 titulaires et 34 adjoints entre le 22 novembre et le $1^{\text {er }}$ décembre 2011.

\section{T1 Conclusion}

Les pharmaciens d'officine éprouvent encore des difficultés à trouver leur place dans les programmes d'éducation du patient, pour la plupart déployés en secteur institutionnel. L'ETP doit être conçue comme un prolongement logique du service rendu au malade chronique, nécessitant une expertise pharmaceutique, une posture d'écoute et d'accompagnement adaptée aux besoins individuels ainsi qu'une mise en réseau des informations sur le patient. Le pharmacien dispose de ces atouts : il doit œuvrer pour être reconnu légitime pour ses connaissances sur le médicament et développer ses compétences pédagogiques ainsi que des moyens de communication actifs avec ses partenaires.

\section{Points à retenir}

- L'observance est capitale chez le patient greffé et passe par une bonne adhésion thérapeutique.

- Outre les immunosuppresseurs, différentes thérapeutiques associées doivent être prises au long cours pour limiter les atteintes du greffon (traitement de l'hypertension, de l'hypercholestérolémie, de l'hyperuricémie...).

- La surveillance des patients transplantés doit être rigoureuse afin de prévenir les complications, notamment infectieuses, cardiovasculaires et cutanées (risque tumoral).

- Le recours à l'automédication doit être très limité, les produits potentiellement néphrotoxiques tels que les anti-inflammatoires non stéroïdiens étant totalement proscrits.

- Une prise en charge globale sur le plan clinique, thérapeutique, économique et psychosocial doit être envisagée. 


\section{Références}

[1] Rabiller J. Exploration des difficultés dans la prise de traitements au long cours chez le patient transplanté rénal : comment le rendre acteur de sa santé. [Thèse de doctorat en pharmacie]. Angers: Université d'Angers; 2013.

[2] Baudrant-Boga M, Lehmann A, Allenet B. Penser autrement l'observance médicamenteuse : d'une posture injonctive à une alliance thérapeutique entre le patient et le soignant. Concepts et déterminants. Annales Pharmaceutiques Françaises. 2012;70(1):15-25.

[3] Hamburg B, Inoff G. Relationships between behavioral factors and diabetic control in children and adolescents: a camp study. Psychosom Med. 1982;44(4):321-39.

[4] Ministère de la Santé, de la Jeunesse, des Sports et de la Vie associative. Plan 2007-2011 pour l'amélioration de la qualité de vie des personnes atteintes de maladies chroniques.

www.sante.gouv.fr/IMG/pdf/plan2007_2011.pdf

[5] Tourette-Turgis $C$, Isnard Bagnis $C$, Pereira P. L'éducation thérapeutique dans la maladie rénale chronique : le soignant pédagogue. Paris: Comment dire; 2008.

[6] Ben Abdelazie A, Gaha K, Mhamdi Y et al. Qualité de la rédaction de l'ordonnance médicale dans les structures de médecine générale. Thérapie. 2005;60(2):117-23.

[7] Halioua B. L'observance thérapeutique dans les traitements locaux du psoriasis. Rev Prat. 2012;62(2):156-9.

[8] Chronisanté Chronicité Santé Société. L'impact des maladies chroniques : les psychologues ont un rôle à jouer. http://chronisante.inist.fr/?2008-L-impact-des-maladies

[9] Chronisanté Chronicité Santé Société. Le rôle du psychologue dans l'éducation thérapeutique du patient en néphrologie. http://chronisante.inist.fr/?2010-Le-role-du-psychologue-dans-I

[10] Miramond M. Le rôle du pharmacien d'officine dans la prise en charge des dyslipidémies : de la sécurisation à l'accompagnement. [Thèse de doctorat en pharmacie]. Grenoble: Faculté de pharmacie de Grenoble; 2012.

[11] www.cespharm.fr

[12] Fournier C, Feldman D, Greffier C et al. Designing a tool for therapeutic patient education about generic drugs: from idea to setting up. Ther Patient Educ. 2011;3(2):101-10.

[13] Agence nationale de sécurité du médicament et des produits de santé (ANSM). www.ansm.sante.fr/Declarer-un-effet-indesirable/Pharmacovigilance/Centres-regionaux-depharmacovigilance/(offset)/4

[14] www.francerein.org

[15] www.renaloo.com

[16] Buxeraud J. Le dossier pharmaceutique. Act Pharm. 2009;48(487):10-7. 
[17] Patients sensibles, entre écoute et confidentialité. La revue Pharma. 2012;88:14-5.

[18] Brunie V, Vignand C. Éducation thérapeutique du patient cardiaque : retour d'expérience à I’hôpital Antoine Béclère. J Pharm Clin. 2010;29(2):98-102.

Déclaration de liens d'intérêts :

Les auteurs déclarent ne pas avoir de liens d'intérêts.

\section{Figures}

\section{Rab_fig1}

Figure 1. Planche de traitements pouvant être remise au patient transplanté rénal.

(c) N. Clere

Sur 1 colonne près de son appel

\section{Rab_fig2}

Figure 2. Exemple de plan de prise médicamenteuse.

(C) N. Clere

Sur 1 colonne + marge près de son appel

\section{Rab_fig3}

Figure 3. Plan de prise pouvant être proposé afin d'aider le patient à s'adapter aux doses dégressives de corticoïdes.

(C) N. Clere

Sur 1 colonne + marge près de son appel 


\section{Rein}

Asthme

\section{Tension}

\section{Cœur}

\section{Cholestérol}




\begin{tabular}{|c|c|c|c|c|c|}
\hline $30 / 01 / 2019$ & $31 / 01 / 2019$ & $01 / 02 / 2019$ & $02 / 02 / 2019$ & $03 / 02 / 2019$ & 04/02/2019 \\
\hline \multicolumn{2}{|c|}{$\begin{array}{l}\text { Cortancyl }{ }^{\circledR} 15 \mathrm{mg} \\
\text { le matin au petit déjeuner : } \\
\text { prendre } 3 \text { comprimés de } 5 \text { mg }\end{array}$} & \multicolumn{2}{|c|}{$\begin{array}{l}\text { Cortancy }{ }^{\circledR} 14 \mathrm{mg} \\
\text { le matin au petit déjeuner : } \\
\text { prendre } 2 \text { comprimés de } 5 \mathrm{mg} \\
\text { et } 2 \text { comprimés de } 2 \mathrm{mg}\end{array}$} & \multicolumn{2}{|c|}{$\begin{array}{l}\text { Cortancyl }{ }^{\circledR} 13 \mathrm{mg} \\
\text { le matin au petit déjeuner : } \\
\text { prendre } 2 \text { comprimés de } 5 \mathrm{mg} \\
\text { et } 3 \text { comprimés de } 1 \mathrm{mg}\end{array}$} \\
\hline
\end{tabular}
prendre 3 comprimés de $5 \mathrm{mg}$ et 2 comprimés de $2 \mathrm{mg}$ et 3 comprimés de $1 \mathrm{mg}$ 